\section{Does intravenous administra- tion of recombinant tissue plasminogen activator for ischemic stroke can cause inferior myocardial infarction?}

\author{
Mostafa Almasi, Saeed Razmeh, \\ Amir Hassan Habibi, Amir Hassan Rezaee \\ Department of Neurology, Rasoul Akram \\ Hospital, Iran University of Medical \\ Sciences, Tehran, Iran
}

\begin{abstract}
Recombinant tissue plasminogen activator (rTPA) is one of the main portions of acute ischemic stroke management, but unfortunately has some complications. Myocardial infarction (MI) is a hazardous complication of administration of intravenous rTPA that has been reported recently. A 78-year-old lady was admitted for elective coronary artery bypass graft surgery. On the second day of admission, she developed acute left hemiparesis and intravenous rTPA was administered within 120 minutes. Three hours later, she has had chest pain. Rescue percutaneous coronary intervention was performed on right coronary artery due to diagnosis of inferior MI, and the symptoms were resolved.
\end{abstract}

\section{Introduction}

The introduction of intravenous alteplase, which is a recombinant tissue plasminogen activator (rTPA) for reperfusion therapy, has led to a dramatic change in approach to patients with ischemic stroke. The intravenous route of this medication should be used in acute phase of ischemic stroke, notably within 4.5 hours of initiation of ictus. ${ }^{1}$ The typical method for administration of intravenous alteplase is the total dosage of $0.9 \mathrm{mg}$ per kilogram of body weight (maximum 90 $\mathrm{mg}$ ) that one tenth of total dosage is administered as a bolus dose and the remaining $90 \%$ of total dosage is infused over the next hour. ${ }^{2}$

However, administration of rTPA had some hazardous dangers. There are some risks of major bleeding, particularly in the brain; therefor, patients need to be carefully selected based on eligible criteria. Hypersensitivity reaction including angioedema, reocclusion and secondary embolization and brain edema due to reperfusion injury are the other reported complications of rTPA administration in ischemic stroke. ${ }^{3}$
Cardiac complications following acute cerebrovascular accidents are common, but fortunately many of them had minimal cardiac adverse effects.4,5 Up to $12 \%$ of acute cerebrovascular accidents (CVAs) may be associated with acute myocardial infarction within 72 hours of onset.4,6 There are some possible explanation, but the clear cause is still unknown. There may be an underlying cardiac embolic source for both ischemic stroke and acute myocardial infarction such as a left ventricular thrombus. ${ }^{7}$ Embolic stroke to insula can cause autonomic dysfunction and cardiac arrhythmia which can cause ischemia of myocardium due to increasing demand of premorbid coronary arteries. ${ }^{8}$ Several conditions such as aortic dissection, large vessel arteritis and endocarditis can induce myocardial infarction and ischemic stroke, simultaneously. ${ }^{9,10}$ In addition, some drugs like amphetamines and cocaine may lead to concurrent occurrence of myocardial infarction and ischemic stroke. 11

An indication of acute myocardial infarction after administration of rTPA for ischemic stroke is increasingly reported.7,9,10 In this study, we described a patient with previous cardiovascular risk factors who was complicated with acute myocardial infarction following prescription of rTPA for an acute ischemic stroke.

\section{Case Report}

A 78-year-old right-handed lady was referred to elective cardiac surgery department for coronary artery bypass graft (CABG) surgery. She was hospitalized for conducting the preoperation investigations. On the second day of admission, she developed sudden onset weakness of left upper and lower limbs and dropping of left side of the face. She has had a past history of hypertension, which was controlled by taking $50 \mathrm{mg}$ atenolol twice a day. She has had angina pectoris but not any history of myocardial infarction. According to her coronary angiography, she was candidate for CABG surgery due to coronary artery stenosis.

The vital signs at the onset of left side hemiparesis included pulse rate of 74/min with regular rhythm, respiratory rate of $16 / \mathrm{min}$, blood pressure of $160 / 100 \mathrm{~mm} \mathrm{Hg}$ and normal body temperature. Her baseline electrocardiogram showed evidences of left ventricular hypertrophy (LVH) and there was no evidence of acute cardiac ischemia.

Axial brain computed tomography (CT) scan without contrast, which has been done after 30 minutes of hemiparesis, showed no evidence of hemorrhage, no new parenchymal hypodensity and no dense clot sign. Although, the brain CT scan showed an old stroke in left hemisphere which was not compatible with the
Correspondence: Saeed Razmeh, Rasoul Akram Hospital, Niayesh St, Sattar-Khan St, Tehran, Iran.

Tel: +98.9151801384 .

E-mail: srazme82@gmail.com

Key words: Recombinant tissue plasminogen activator; Myocardial infarction; Acute ischemic stroke.

Contributions: all of the authors contribute in gathering the information of the patient. The diagnosis and management of patient was conducted by SR and AHH. MA, SR and AHH participate in writing and editing of manuscript.

Conflict of interest: the authors declare no poten tial conflict of interest.

Received for publication: 22 May 2016 Accepted for publication: 30 May 2016.

This work is licensed under a Creative Commons Attribution NonCommercial 4.0 License (CC BYNC 4.0).

(C) Copyright M. Almasi et al., 2016

Licensee PAGEPress, Italy

Neurology International 2016; 8:6617

doi:10.4081/ni.2016.6617

symptoms of the patient. Due to acute left hemiparesis and no evidence of hemorrhage on brain CT scan, the diagnosis of ischemic stroke with unclear mechanism was made. The National Institutes of Health Stroke Scale (NIHSS) was 10 out of 42 , composed of 2 score for partial paralysis of lower face, 3 score for left arm motor weakness, 3 score for left leg motor weakness, 1 score for sensory disturbances and 1 score for language problem. Next decision was to administer the intravenous thrombolytic. Intravenous rTPA was started after 120 minutes of the event onset. The bolus dosage of rTPA was $8 \mathrm{mg}$, followed by infusion of $58 \mathrm{mg}$ in the next 60 minutes.

Approximately three hours later, the patient complained of chest pain and palpitation. The immediate electrocardiogram (ECG) showed ST-segment elevations in the inferior leads (II, III, aVF), and ST-segment depressions in the anterolateral leads (I, aVL) (Figure 1). The patient was emergently transferred to the cardiac catheterization laboratory for rescue percutaneous coronary intervention (PCI). On angiography, the occlusion of right coronary artery was detected. Thrombectomy via an aspiration catheter was performed and normal coronary blood flow was restored. Then PCI was conducted on right coronary artery and posterior descending artery bifurcation with two bare metal stents. After revascularization, the patient's heart rhythm returned to normal sinus and hypotension was resolved. The brain CT scan was repeated and showed a new hypo- 


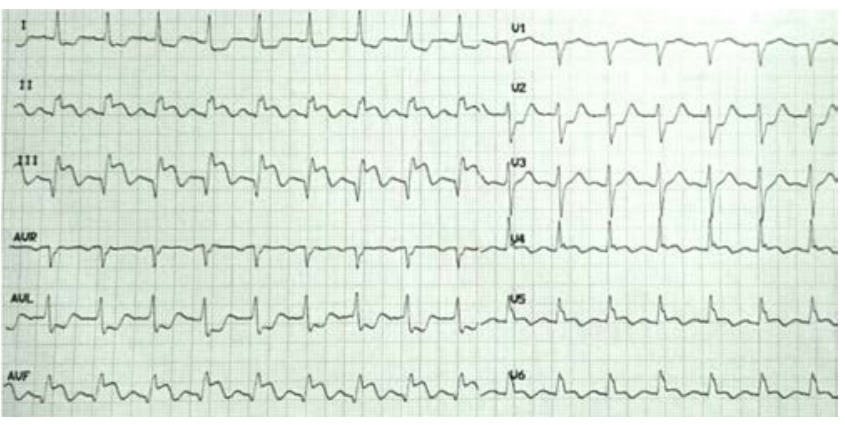

Figure 1. 12-lead-electrocardiogram showing ST-segment elevations in the inferior leads (II, III, aVF), and ST-segment depressions in the anterolateral leads (I, aVL).

density in right parietal lobe. Brain MRI with diffusion-weighted sequence (DW) revealed a watershed ischemic infarction between anterior cerebral artery (ACA)-multiple correspondence analysis (MCA) and MCA-principal component analysis (PCA) territories in right side, which was hyposignal in equivalent Apparent Diffusion Coefficient (ADC) sequence that means an acute stroke occurred (Figure 2). The echocardiography revealed a left-ventricular ejection fraction of $50 \%$ with inferior hypokinesia and without evidence of intracardiac thrombus or intracardiac right-to-left shunt.

\section{Discussion}

In this case, the development of MI related to the use of IV rTPA within 24 hours after receiving of IV rTPA was presented. Despite having previous major involvement of coronary arteries, the patient had not any symptoms of acute myocardial infarction before admission and during the first day of admission. Sudden occurrence of acute left hemiparesis in our patient may have some possible mechanisms; first, the coronary artery disease may be associated with diffuse atherosclerosis, which involved carotid artery simultaneously. The other possible explanation is an embolus originating from the morbid heart or a plaque of aorta. In fact, the both of thrombotic or embolic mechanism for ischemic stroke may be the presumed mechanism, but the evidence of an old stroke in left hemisphere and now incidence of another stroke in left hemisphere signifies the embolic mechanism compared to thrombotic mechanism.

Our patient developed a myocardial infarction in right heart after administration of rTPA. One can suppose that there was a cardiac clot, which was the mechanism of ischemic stroke and itself introduced an extra embolus to right coronary artery after the injection of rTPA. The presence of cardiac clot

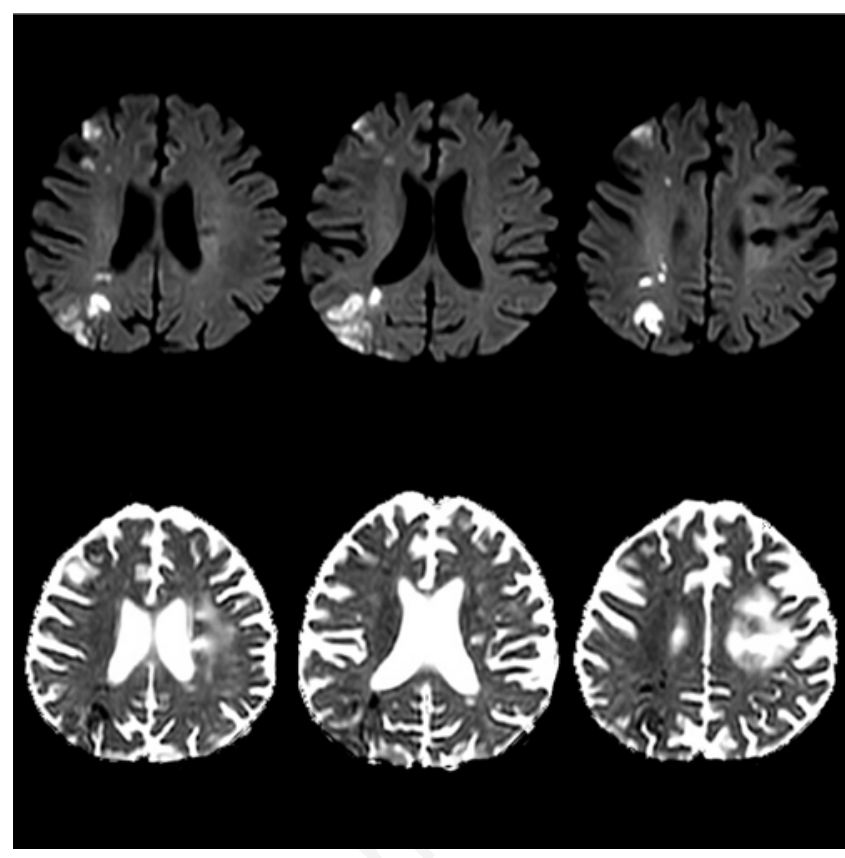

Figure 2. Axial view of brain magnetic resonance imaging, diffusion-weighted and apparent diffusion coefficient sequence: watershed acute ischemic infarction between apparent diffusion coefficient - multiple correspondence analysis and multiple correspondence analysis - principal component analysis territories in right side; hypersignality in diffusion-weighted and hyposignality in apparent diffusion coefficient is revealing water restriction in acute ischemia. The hypersignal area in apparent diffusion coefficient sequence in left hemisphere is related to an old stroke. during ischemic stroke can be 10 to $15 \%$ based on the used modality including transesophageal echocardiography. ${ }^{12,13}$ In fact rTPA can degrade the clot to small fragments with subsequent embolization to coronary arteries leading to MI. ${ }^{9}$

The presented patient afflicted with an inferior MI after administration of rTPA. In the cases that presented with Stewa et al. ${ }^{9}$ and Wallace et al., ${ }^{7}$ also the patients developed an inferior MI following administration of rTPA. It is not clear if there is any direct correlation between rTPA injection and occurrence of inferior MI, or maybe there is an identical etiology for both of ischemic stroke and inferior MI, as we encounter in aortic dissection. Contraries, there are some evidences for development of ischemic stroke as a consequence of inferior MI.14,15 In addition to common cause of ischemic stroke after any types of MI, the inferior MI has some special characteristics which are risk factors for ischemic stroke. Inferior MI can lead to infarction of right ventricle in about half of the cases, which is associated with severe hemodynamic compromise and diminished cardiac output. ${ }^{14}$ Hemodynamic compromise decrease cerebral perfusion especially in premorbid vessels and lead to stroke. It is also shown that inferior MI can be complicated with transient atrial fibrillation and future risk of ischemic stroke in preserve of ejection fraction will increased. 15

\section{Conclusions}

Nowadays rTPA is widely used for the treatment of acute stoke. The occurrence of MI especially inferior MI had been reported following administration of rTPA but current data cannot clarify the underlying reason. It is a hazardous complication that needs to be carefully considered after administration of rTPA and should be treated quickly.

\section{References}

1. Song S. Hyperacute management of ischemic stroke. Semin Neurol 2013;33: 427-35.

2. Sharma VK, Ng KW, Venketasubramanian $\mathrm{N}$, et al. Intravenous thrombolysis for acute ischemic stroke in Asia. Expert Rev Neurother 2012;12:209-17.

3. Balami JS, Sutherland BA, Buchan AM. Complications associated with recombi- 
nant tissue plasminogen activator therapy for acute ischaemic stroke. CNS Neurol Disord Drug Targets 2013;12:155-69.

4. Hacke W, Kaste M, Fieschi C, et al. Randomised double-blind placebo-controlled trial of thrombolytic therapy with intravenousalteplase in acute ischaemic stroke (ECASS II). Second EuropeanAustralasian Acute Stroke Study Investigators. 1998;352:1245-51.

5. Reuter H, Erdmann E. [Elevation of the ST segment and positive troponin $\mathrm{T}$ as a sign of myocardial damage in acutestroke]. Dtsch Med Wochenschr 2009;134:2333-6. [Article in German]

6. Chin PL, Kaminski J, Rout M. Myocardial infarction coincident with cerebrovascular accidents in the elderly. Age Ageing 1977;6:29-37.

7. Wallace EL, Smyth SS. Spontaneous coro- nary thrombosis following thrombolytic therapy for acute cardiovascular accident and stroke: a case study. J Thromb Thrombolysis 2012;34:548-51.

8. Abboud H, Berroir S, Labreuche J, et al. Insular involvement in brain infarction increases risk for cardiac arrhythmia and death. Ann Neurol 2006;59:691-9.

9. Sweta A, Sejal S, Prakash S, et al. Acute myocardial infarction following intravenous tissue plasminogen activator for acute ischemic stroke: an unknown danger. Ann Indian Acad Neurol 2010;13:64-6.

10. Mehdiratta M, Murphy C, Al-Harthi A, Teal PA. Myocardial infarction following t-PA for acute stroke. Can J Neurol Sci 2007; 34:417-20.

11. Fonseca AC, Ferro JM. Drug abuse and stroke. Curr Neurol Neurosci Rep 2013;13:325.
12. Lee RJ, Bartzokis T, Yeoh TK, et al. Enhanced detection of intracardiac sources of cerebral emboli by transesophageal echocardiography. Stroke 1991;22:734-9.

13. Eriksson S, Backman C, Osterman G. Pulmonary-artery cineangiocardiography and echocardiography for detection of cardiac sources of cerebral embolism. Acta Med Scand 1988;223:27-33.

14. Goldstein JA. Right heart ischemia: pathophysiology, natural history, and clinical management. Prog Cardiovasc Dis 1998; 40:325-41.

15. Siu CW, Jim MH, Ho HH, et al. Transient atrial fibrillation complicating acute inferior myocardial infarction: implications for future risk of ischemic stroke. Chest 2007;132:44-9. 